\title{
A CLINICAL PLAN FOR MDMA (ECSTASY) In THE TREATMENT OF POST-TRaumatic StRESS DisORDER (PTSD): PARTNERING WITH THE FDA
}

\author{
Rick Doblin, Ph.D.
}

The following article was originally published in the April-June 2002 special MDMA issue of the Journal of Psychoactive Drugs (www.hafci.org). The article presents the rationale behind MAPS' efforts to sponsor research in Spain, the US and Israel investigating MDMA's potential in treating patients suffering from posttraumatic stress disorder (PTSD). This document is the clearest expression to date of MAPS' role as a membership-based non-profit pharmaceutical company, as distinct from MAPS' other research and educational functions. We are reprinting this article in order to explain in detail to MAPS' membership the vision and strategy animating MAPS' MDMA/PTSD research projects and associated fundraising efforts. A mission statement in a way, this article should help to explain why MAPS has chosen the ambitious goal of developing MDMA into an FDA-approved prescription medicine in the treatment of PTSD. Since this article was written, the Spain MDMA/PTSD research project has been halted (hopefully temporarily) due to political pressure, and it has taken longer than expected to obtain DEA and IRB permission to start the US MDMA/PTSD project.

The Multidisciplinary Association for Psychedelic Studies (MAPS, www.maps.org), a membership-based non-profit research and educational organization, is sponsoring a series of studies designed to develop MDMA into an FDA-approved prescription medicine, initially for the treatment of post-traumatic stress disorder (PTSD). MAPS is currently sponsoring a pilot MDMA dose-escalation study in Madrid, Spain with PTSD patients, conducted under the direction of Dr. Pedro Sopelana and Jose Carlos Bouso, Ph.D. candidate (Sopelana \& Bouso 1999). This is the world's only ongoing study of the efficacy of MDMA-assisted psychotherapy. On November 2, 2001, a MAPSsponsored study under the direction of Dr. Michael Mithoefer was approved by the FDA, with Institutional Review Board (IRB) approval in process (Mithoefer \& Wagner 2001). MAPS is also working to sponsor an MDMA/PTSD study in Israel, under the direction of Dr. Moshe Kotler.

This paper elaborates a five-year, $\$ 5$ million Clinical Plan outlining a proposed sequence of studies to investigate MDMA-assisted psychotherapy in the treatment of PTSD. This Clinical Plan starts with pilot studies and concludes with two FDA-required "adequate and well controlled investigations" of safety and efficacy. This discussion outlines a strategy for developing MDMA into an FDA-approved prescription medicine. A series of regulatory, ethical and methodological issues for the investigation of psychedelic psychotherapy in the context of FDA-approved clinical trials, which form the basis for the Clinical Plan, are discussed in detail in the context of my Public Policy dissertation (Doblin 2001).

Given the political and scientific hurdles, a rational analysis of the likely return on investment would probably not inspire any venture capitalists to invest their risk capital into the development of MDMA as a prescription medicine. MDMA is off patent, PTSD or any other psychological disorder for which MDMA might be effective affect more than 200,000 people so that patent protection under FDA's Orphan Drug program cannot be obtained, and the political hurdles due to MDMA's non-medical use may not be surmountable within any time frame that an investor 


\section{"This paper elaborates a five-year, \$5 million Clinical Plan outlining a proposed sequence of studies to investigate MDMA-assisted psychotherapy in the treatment of PTSD, as part of a strategy for developing MDMA into an FDA- approved prescription medicine."}

would consider realistic. Though the for-profit approach for the development of MDMA as a prescription medicine is of questionable viability, the non-profit approach is more likely to succeed. There are probably enough philanthropists who, from personal experiences or otherwise, appreciate the political, scientific and medical importance of supporting the struggle to develop MDMA into a legal prescription medicine.

This discussion begins by evaluating the strategic advantages associated with the conduct of FDA-approved research with MDMA for PTSD, as compared to other psychedelics that could be used for psychotherapy and other potential patient populations. Proposed protocol designs and sample sizes for the studies evaluating the potential use of MDMA in the treatment of PTSD are based in part on a review of documents pertaining to Pfizer's successful development of Zoloft into the first FDA-approved medicine for the treatment of PTSD. These documents were obtained from FDA by the author through Freedom of Information Act (FOIA) request. A FOIA request for FDA documents related to its approval of Paxil for PTSD is still pending.

\section{Choosing Drug and Patient Population}

The primary strategic issue in conducting psychedelic psychotherapy research is estimating the probabilities of success in the FDA drug development and approval process of the numerous combinations of any of the psychedelic drugs and patient populations. Psychedelic drugs, though each with a unique set of actions and side effects, all serve the generally similar function of increasing access to psychological, emotional processes. As a result, psychedelics can be used as general purpose adjuncts to psychotherapy, in the treatment of many conditions for which people seek out psychotherapy or psychiatric treatment. The limited resources available to fund psychedelic psychotherapy research make it essential to chose the best test case of a specific psychedelic drug used in treating a specific clinical indication.

\section{Why MDMA?}

On the one hand, the psychological safety profile of MDMA is superior to that of all the other psychedelics. MDMA is relatively short acting with primary effects lasting only about 4 hours with gradual return to baseline over the course of another 2 hours or so. MDMA rarely interferes with cognitive functioning or perception and usually produces a warm, emotionally grounded feeling with a sense of self-acceptance, and a reduction of fear and defensiveness. Subjects under the influence of MDMA can usually "negotiate" with their emergent psychological material and often retain the ability to move at will toward or away from certain thoughts or emotions. In contrast, LSD lasts 8 to 10 hours, interrupts rational cognitive processes, impacts perception, requires surrender to inner emotional processes rather than permitting negotiation, and can result in feelings of loss of control, fear and panic, as well as more positive emotions. All the major psychedelics such as psilocybin, mescaline, ibogaine, DMT, etc., resemble LSD 
more so than they resemble MDMA. Even the effects of marijuana are more similar to the classic psychedelics than to MDMA.

In terms of therapeutic potential, MDMA is remarkable effective, gentle yet profound. Because it operates on emotions more so than cognitive processing, the MDMA state is only subtly different than normal. As a result, the thoughts and emotions of the MDMA state can be easily remembered after the effects of the drug have worn off, facilitating integration and long-term growth. Due to its relative short-acting duration and its gentle action, MDMA has the greatest opportunity of any psychedelic to be integrated into psychiatric practice. The classic psychedelics can be equally or even more therapeutic but in different ways and with greater personal struggles required of patients and therapists.

On the other hand, the physiological safety profile of all the classic psychedelics is superior to that of MDMA. The extreme position on risk is expressed by Dr. Alan Leshner, ex-Director of the National Institute on Drug Abuse (NIDA), who claims that "There is no safe way to use any of these drugs [such as MDMA]," (Mertl 2000) that "even experimenting with club drugs [such as MDMA] is an unpredictable and dangerous thing to do," and that chronic use of MDMA may cause long-term problems with emotion, memory, sleep and pain (Leshner 2001).

When used recreationally in dance clubs, some users of MDMA (mostly in combination with other drugs) have died from hyperthermia as a result of overheating from vigorous dancing in high ambient temperature environments with inadequate water or other fluid replacement. From 1994 through 1999, there have been a total of 68 MDMA-related deaths (may or may not be causal) reported to the Drug Abuse Warning Network (DAWN), though not all deaths were related to hyperthermia. Medical Examiner data reports 1 death associated with MDMA in 1994, 6 in 1995, 8 in 1996, 3 in 1997, 9 in 1998, 41 in 1999 (Office of Applied Studies, 2001a). Rela- tively few of the Medical Examiner cases were for MDMA alone. Most were associated with MDMA used in combination with one or more other drugs. The Medical Examiner numbers do not reflect national totals, which do not exist, but are simply the totals reported by the Medical Examiner offices that are included in the DAWN system. MDMA-related (though not necessarily causally related) hospital Emergency Room visits reported to DAWN (these are national estimates) totaled 247 in 1994, 422 in 1995, 319 in 1996, 637 in 1997, 1142 in 1998, 2850 in 1999, and 4511 in 2000. (Office of Applied Studies, 2001b).

Furthermore, with the exception of ibogaine, the classic psychedelics have not been claimed to be "neurotoxic," as has MDMA. In primates, at doses slightly higher than the amounts used in psychotherapy, MDMA has been linked to minor persisting reductions in serotonin levels in a few brain regions (Ricaurte et al. 1988), with the no-effect level for serotonin reductions in primates being $2.5 \mathrm{mg} / \mathrm{kg}$, administered orally once every two weeks for four months (8X) (Ricaurte, unpublished, cited in Vollenweider et al. 1999a). Whether therapeutic doses of MDMA have any permanent impact on serotonin levels is a matter of substantial controversy (Lieberman \& Aghajanian 1999). If high doses of MDMA are consumed frequently, a dosage pattern seen in some recreational users of MDMA, MDMA may reduce serotonin levels for extended periods of time (McCann et al. 1998). Though there is evidence of recovery of serotonin levels over time, serotonin does not reach initial levels in all brain regions while some brain regions recover to levels higher than baseline (Fischer et al. 1995). Some changes may be permanent (Hatzidimitriou, McCann \& Ricaurte 1999). Fortunately for the heavy recreational users of MDMA, these changes in serotonin levels, if they do indeed occur in humans, seem largely asymptomatic. Evidence for any functional consequences in animals or humans resulting from even massive consumption of MDMA is 
weak. Concern centers around a series of studies that show statistically significant but mostly clinically insignificant reductions in a few memory functions in heavy poly-drug users who have consumed large amounts of MDMA (Bolla, McCann and Ricaurte 1998; Reneman et al. 2001; Zakzanis \& Young 2001; Croft et al. 2001; Gouzoulis-Mayfrank et al. 2000; Gamma 2001). Concerns that negative functional consequences associated with MDMA use will increase over time as MDMA users age are hypothetical, and are not evidence-based.

Research has shown that neurotoxicity is exacerbated by high body temperatures and can be eliminated by a slight cooling of body temperature (Malberg, Sabol \& Seiden 1996; Malberg \& Seiden 1998). The effect of temperature makes data about risk that is gathered from people who take MDMA at raves of limited predictive value for estimating the risk of subjects exposed to MDMA in clinical settings. MDMA's increased risk profile is a direct result of its use in recreational settings, with use in clinical research settings relatively non-problematic (Vollenweider et al. 1999a). In therapy, MDMA is not used on a daily basis but rather as an adjunct to psychotherapy administered a relatively few times, with several weeks between therapy sessions. The most sophisticated investigation of MDMA-neurotoxicity has been conducted by Dr. Franz Vollenweider at the U. of Zurich. Dr. Vollenweider found no evidence for serotonin reductions in MDMA-naive subjects who were given a PET scan shortly before and then again four weeks after receiving a moderate amount of MDMA in the therapeutic dose range $(1.5-1.7 \mathrm{mg} / \mathrm{kg}$ ) (Vollenweider 2001).

The combination of the remarkable therapeutic potential of MDMA, along with its substantial safety for use in clinical settings, makes it a very attractive choice for drug development. A comprehensive risk/benefit analysis that lent support to the case for clinical psychotherapy research with MDMA was funded by MAPS and submitted to FDA (Baggott, Jerome \& Stuart,
2001). Politically, however, MDMA is not the easiest psychedelic to try to develop into a prescription medicine. Its non-medical use is increasing, especially among young people. In the 2001 Monitoring the Future survey, funded by NIDA, $11.7 \%$ of high school seniors reported that they had tried Ecstasy at some point, up from $11.0 \%$ in 2000 and $8.0 \%$ in 1999 (Johnston, 0'Malley \& Bachman 2001). Police authorities are seizing increasingly large amounts. Customs officials have seized 9.3 million ecstasy pills in FY 2000, as compared to 3.5 million in FY 1999 and 750,000 in FY 1998 (Office of Public Affairs, US Customs Service, 2000; National Drug Intelligence Center, 2000). NIDA has called the increased use of MDMA an epidemic (NIDA 2000).

Yet the political controversy about MDMA offers one crucial advantage that makes MDMA much more likely to become the first psychedelic to be approved as a prescription medicine. As a result of the millions of non-medical users of MDMA around the world, health authorities, anti-drug authorities and research scientists have expended an amazing amount of time, energy and money trying to understand the risks of MDMA, its mechanisms of action, and the consequences of acute and long-term use.

The number of scientific papers in the peerreviewed scientific literature reporting on research with MDMA in humans and animals, along with case reports discussing adverse events, exceeds 1240 according to a Medline search conducted by the author on May 1,2002. Data in the peer-reviewed scientific literature can be submitted to FDA as evidence in the assessment of MDMA's risk profile and safety, with the only cost being the time it takes to systematically review the papers and organize the data for submission to FDA. FDA is willing to accept published papers for review and has even approved drugs "based primarily or exclusively on published reports (FDA 1998)." The costs of conducting these published MDMA studies is well over $\$ 20$ million. The availability of data from 
these studies dramatically reduces the amount of additional funding that will be required to argue a case before FDA for MDMA's safety and efficacy.

Several researchers have administered MDMA to human subjects in clinical studies of MDMA's safety, mechanism of action and physiological and psychological effects. More frequently, researchers have compared people who have used MDMA in non-medical contexts with controls. As of March 2002, more than 262 subjects had been administered MDMA in the context of legal research. There was also data in the scientific literature from more than 985 people who had used MDMA, sometimes in astonishingly large amounts, in non-medical recreational contexts. These MDMA users have been compared to more than 835 controls."

An MDMA Phase I study with 18 patients has been successful completed in the United States, though data on only the first 6 subjects have been published (Grob et al. 1996). Two other Phase I studies with MDMA focused on objectives other than safety have also been conducted in the United States. An MDMA pharmacokinetic study was conducted at UC San Francisco (Everhart et al. 1999) and a study is underway investigating which brain neurotransmitter receptor sites are involved in producing MDMA's subjective effects (Tancer \& Johanson 2001). Studies in Switzerland have investigated MDMA's action on brain neurotransmitter receptor sites (Liechti et al. 2000), on information processing (Vollenweider et al. 1999b) and on the psychological and cardiovascular effects of a single dose of MDMA (Vollenweider 1998). Three MDMA pharmacokinetic studies have been conducted in Europe in England (Fallon et al. 1999), Spain (de la Torre et al. 2000), and Switzerland (Helmlin et al. 1996). A Phase I dose-response safety study has been completed place in Spain
(Mas et al. 1999; Cami et al. 2000), as well as a study investigating MDMA/alcohol interactions (Hernandez-Lopez et al. 2002). A study investigating the hormonal effects of MDMA has taken place in England (Henry et al. 1998) and a study investigating the immunological effects has taken place in Spain (Pacifici et al. 1999). Yet with all this research, there is not one single paper reporting data from a controlled scientific study into the therapeutic use of MDMA.

MAPS' effort to initiate controlled, FDAapproved scientific research into the therapeutic potential of MDMA in patient populations began in 1986, and has taken 16 years to come to fruition. A Phase II dose-escalation pilot study of MDMA-assisted psychotherapy in the delic drugs, though each with a unique set of actions and side effects, all serve the generally similar function of increasing access to psychological, emotional processes. As a result, psychedelics can be used as general purpose adjuncts to psychotherapy."

treatment of post-traumatic stress disorder (PTSD) has been approved in Spain. This is currently the only study into the therapeutic use of MDMA approved anywhere in the world. The existence of the Spain study, sponsored by MAPS, is an important practical factor behind the selection of MDMA as the initial psychedelic drug to focus on developing into an FDA-approved prescription medicine. The sixth patient in Spain, at the $75 \mathrm{mg}$. dose level, was treated on April 15,2002 . The researchers conducting the study will gather the data in a sufficiently rigorous manner so that it can be submitted to FDA for review. With the approval of this study, the chance to develop the therapeutic potential of MDMA is now more than a mirage. 


\section{Why Post-Traumatic Stress Disorder?}

In choosing the patient population to study, one of the criteria was that the unique properties of MDMA-enhanced psychotherapy needed to be matched to a patient population in which MDMA therapy could offer a dramatic benefit. Ideally, this benefit would require only from one to three drug sessions to produce significant, measurable and long-lasting clinical progress. Alternative medications for this patient population should be relatively ineffective, at least in some subpopulation of patients. The patient population should also be a group that the general public feels compassion towards, in order to help overcome resistance to the idea of the therapeutic use of psychedelics.

The core of the MDMA experience has been described by one of the pioneering psychiatrists who worked with MDMA-assisted psychotherapy in terminal cancer patients as "reducing the fear response to a perceived emotional threat." When used therapeutically, MDMA is administered as an adjunct to psychotherapy on an intermittent basis within a larger therapeutic relationship, usually fewer than four times and frequently only once or twice. Numerous case histories and anecdotal reports testify to MDMA's ability to assist people struggling to come to terms with difficult life events (Stevens 1999/2000; Otalora 1984). These reports suggest that MDMA-assisted psychotherapy should initially be explored not in patients whose psychiatric symptoms originated with biological imbalances with possible genetic components, though MDMA might still be helpful in some ways with such patients, but rather in patients who need some assistance in processing difficult emotions that have a deep component of fear and/or anxiety. Two of the main categories of patients that fit this description are people suffering from PTSD and people facing terminal illness. People with these two types of clinical conditions have been treated with MDMA with some remarkable results in some patients.
The main advantage of working with a PTSD patient population instead of patients with terminal illness is that PTSD patients as a group are probably in better overall health than cancer patients and are taking fewer other medications, making it less complicated to work with them. Once the MDMA/PTSD study is underway in the US, MAPS will seek to obtain FDA approval for a study of MDMA-assisted psychotherapy in hospice patients.

In the US market, there are only two conventional pharmacological treatments that have been approved for patients with PTSD. On December 7, 1999, FDA approved the drug known as Zoloft (sertraline) for PTSD, on the basis of four small clinical trials (it was already on the market as an anti-depressant). Two of the clinical trials showed no efficacy, two showed some efficacy. These studies involved a total of 351 subjects. Subgroup analysis revealed that Zoloft was efficacious in female patients but not in male patients. According to Dr. Katz, Director of the Division of Neuropharmacological Drug Products, "The effect of the treatment appears to come essentially completely from women (Katz 1999)." On December 14, 2001, FDA approved the use of Paxil (paroxetine) in the treatment of PTSD. Unlike the Zoloft trials, studies with Paxil showed efficacy in both men and woman. Interestingly, Zoloft and Paxil's mechanism of action is to increase the amount in the synapse of the brain neurotransmitter serotonin, the same neurotransmitter that MDMA primarily impacts. The difference is that MDMA increases serotonin acutely for a period of 4-8 hours after a single dose while Zoloft increases serotonin chronically but must be taken on a daily basis.

The patient group that will be tested with MDMA in Spain is women survivors of sexual assault who suffer from chronic PTSD and who have already failed on at least one course of conventional treatment. The patient group that will be tested in the US will include men and women, survivors of sexual and/or criminal assault, who have failed on one course of treat- 
ment with an SSRI such as Zoloft or Paxil. The patient group for the Israeli study will include patients who have PTSD as a result of war or terrorism, as well as sexual or criminal assault, and who have failed on one course of an SSRI. By choosing subjects who have already failed on one course of conventional treatment, the risk/benefit ratio is improved in favor of permitting the study to proceed.

We hypothesize that MDMA will prove helpful in resolving some of these subjects' difficult and painful memories so that they can move forward with some degree of resolution, not forgetting the past but not as burdened by it either. MDMA-assisted psychotherapy also has the potential advantage of being cost-effective, since it can be delivered within a relatively short time.

MDMA in the treatment of PTSD is probably the best combination of psychedelic drug and clinical indication that can most justify a focused drug development effort. What such a drug development plan might look like will be elaborated below, after a brief review of the discussion of the Psychopharmacologic Drugs Advisory Committee that recommended that Zoloft be approved for use in the treatment of PTSD.

\section{FDA Review of Zoloft for PTSD}

Pfizer's recent experience with its successful development of Zoloft for the treatment of PTSD offers the most direct window into FDA policies and procedures for the design of research protocols and the review of data for the pharmacological treatment of PTSD. There are many analogous issues and also important differences between the development of Zoloft, a medication that has been approved by FDA for daily use for the relief of symptoms associated with PTSD, and the development of MDMA, a drug that is meant to be administered from 1-3 times on an in-patient basis as an adjunct to psychotherapy for the relief of the underlying causes of PTSD. The public record related to FDA ap- proval of Zoloft will be reviewed in order to understand FDA regulatory policy as it applies directly to the development of medications to treat PTSD. The most valuable documents in the public record include transcripts of the 0 ctober 8 , 1999 Psychopharmacologic Drugs Advisory Committee (Psychopharmacologic Drugs Advisory Committee 1999), a slide show delivered at that meeting by Dr. David Smith, Statistical Reviewer, FDA Office of Biostatistics (Smith 1999), and a complete file of the FDA approval package for Zoloft, NDA19839,S026, obtained from FDA through Freedom of Information Act (FOIA) request. As of May 2002, FDA has not yet responded to a FOIA request for the Paxil approval package.

\section{October 8, 1999 Psychopharmacologic Drugs Advisory Committee Meeting: Study Design Issues}

Four clinical trials were reviewed on 0ctober 8, 1999 by FDA's Psychopharmacologic Drugs Advisory Committee, advising the Division of Neuropharmacological Drug Products. Outcome data was presented at the meeting by Pfizer and FDA representatives.

The Advisory Committee meeting began with an overview presented to the Committee by Dr. Tom Laughren, Team Leader for Psychopharmacology at FDA. He indicated that PTSD is a chronic disorder and FDA, "ordinarily uses parallel group studies although one might ask whether a crossover design might be appropriate even for a chronic condition, if the condition is very stable over time and there is a return to baseline if the treatment is stopped (Psychopharmacologic Drugs Advisory Committee 1999: 10)."

Dr. Laughren further noted that, "this is a chronic disorder and one may ask the question whether or not there is a need for long-term data and at what point in development should that information become available should that become an issue for approvability. Now, as an 


\section{"There are many analogous issues and also important differences between the development of Zoloft...and the development of MDMA."}

aside, I should say that we never, up until now, made that a requirement for approving a new indication in psychiatric disorders (Psychopharmacologic Drugs Advisory Committee 1999: 11)."

Dr. Farfel, a Pfizer scientist, indicated that "subjects were dosed once daily beginning with $25 \mathrm{mg} / \mathrm{dy}$ in the first week [dosing was not initially based on $\mathrm{mg} / \mathrm{kg}$ ] and then continuing flexibly titrated between 50 and $200 \mathrm{mg} / \mathrm{dy}$ thereafter (Psychopharmacologic Drugs Advisory Committee 1999: 33)." FDA's Dr. Temple commented about the titration design, indicating that he would have preferred fixed doses. He said, "I would be curious as to why that design was chosen. If it was chosen to avoid adverse effect, that would make some sense, but ordinarily I think you would learn more from a randomization to fixed doses, even if you inched your way up to those doses... Now you could analyze this to see if there was a dose/response hidden in Psychopharmacologic Drugs Advisory Committee 1999: 127)." Dr. Hammer, Advisory Committee member, made the suggestion that one of the major studies should have been fixed dose and the other flexible, so as to have gained some information about dose/response relationships in one of the studies.

Dr. Laughlen said, "One thing that we like to see for an indication that is more mature in some sense than this is, from a regulatory standpoint, we like to see an active control arm in a trial to help us in interpreting it, so that if an active standard drug, which is believed to work, also fails, we are more inclined to discount that study. This is obviously not a strategy you can use early on in the development of a new indication (Psychopharmacologic Drugs Advisory Committee 1999: 145)."

This suggestion of an active control arm for subsequent treatments for PTSD should be adopted. In testing MDMA-assisted psychotherapy for PTSD, parallel groups are more appropriate than a crossover design since the hypothesis is that there will not be a return to baseline after the MDMA treatment is over. This is different for Zoloft, which offers mostly symptomatic relief with a significant number of subjects relapsing once the use of Zoloft is ended. From a financial perspective, this seems ideal for a pharmaceutical company since patients have a continued need to purchase the product or the symptoms will return. In contrast, MDMAassisted psychotherapy has been helpful in some reported case histories after one to three sessions, with no additional MDMA sessions required to maintain clinical improvement.

The fact that the Zoloft design allowed titration suggests that it might also be possible to titrate the number of doses of MDMA-assisted psychotherapy a patient receives in one of the Phase III trials, to match the treatment to the depth and speed at which the patient is able to resolve issues related to the original trauma.

\section{Sample Size for Efficacy}

Dr. Gary Ryan, Group Director of Clinical Research, Pfizer, stated, “Our PTSD Clinical Trial program consisted of four placebo controlled trials enrolling a total of 757 patients (Psychopharmacologic Drugs Advisory Committee 1999: 16)." Though Dr. Ryan reported a total of 757 patients, the data presented in the slides by Dr. Smith indicated only 597 subjects, with the difference due to attrition. Pfizer's Dr. Farfel reported that, "the mean number of subjects in each treatment group was approximately 95 , for a total of 376 subjects treated with sertraline and 381 treated with placebo (Psychopharmacologic Drugs Advisory Committee 1999: 32)." 
In the two clinical trials that demonstrated efficacy, a total of 385 patients were enrolled, 191 who received Zoloft and 194 who received placebo (Smith Slide \#9). Dr. Charles Marmar, Professor and Vice Chairman, Department of Psychiatry, UC San Francisco, spoke for Pfizer and noted that "you can see that for the most part the effects, while meaningful, have been modest (Psychopharmacologic Drugs Advisory Committee 1999: 29)," indicating that sample sizes may need to be fairly large, especially in a comparison study between MDMA and Zoloft or Paxil.

Dr. Katz, Director of the Division of Neuropharmacological Drug Products, stated, "There are conditions where we have considered studies positive or approved drugs on the basis of fairly small studies, but in which the treatment has been statistically significantly different from the control. Of course, the smaller the study, the more likelihood that there is some bias creeping in or that there is an imbalance is an important characteristic that you don't really know how to test for, you don't even know what they are necessarily. So we like to see larger studies but there is no specific requirement for numbers (Psychopharmacologic Drugs Advisory Committee 1999: 149)."

\section{Sample Size for Safety}

Dr. Laughren mentioned that "this program overall was relatively small, and so in making a judgement about the safety of Zoloft, we relied heavily on the safety experience on other populations. So, a question is, is that a reasonable extrapolation? (Psychopharmacologic Drugs Advisory Committee 1999: 14) " Dr. Farfel commented on safety reporting, "Safety was investigated in 757 subjects, and nothing that was found in this development program suggests a risk that has not already been identified in previous trials and indications, and is already not described in the labeling (Psychopharmacologic Drugs Advisory Committee 1999: 55)."

The minimal number of MDMA-assisted psy- chotherapy sessions that will be administered to subjects, along with all the safety data already gathered about MDMA from clinical trials around the world, may enable the safety of MDMA in PTSD patients to be investigated with as few subjects as were used in the studies of Zoloft in the treatment of PTSD. This is a reasonable assumption that would change depending on the strength and clarity of the data actually gathered in the clinical studies.

\section{Estimates for Sample Sizes for the MDMA Phase III Trials}

Based on FDA's review of research into the use of Zoloft in the treatment of PTSD, the power of Pfizer's studies as designed was considered inadequate for subgroup analysis but adequate for group comparisons. The studies as completed had roughly 75 subjects per group. According to Dr. Farfel, the groups had a mean initial enrollment of about 95 subjects, with about 75 per group completing the trial and included in final data analysis.

Until the effect size and variance of response to MDMA-assisted psychotherapy is determined, sample sizes cannot be estimated with accuracy. The more pronounced the treatment effect and the smaller the variation in outcomes, the smaller the sample size needs to be to generate significant results (Friedman, Furberg \& Demets 1985). In order to reduce variance so as to reduce sample size, a homogenous patient population with a relatively uniform response should be selected. In the Zoloft studies, there was a substantial difference in response between men and women. The Phase III MDMA studies should be able to avoid this problem through the review of data gathered in the Phase II trials that will evaluate the effectiveness of MDMA in men and in women. The Phase III trials can then be designed either with all men, all women, or a combination. With an advantage in uniformity over the Zoloft designs, it will probably be possible to obtain adequate power with 80 sub- 
jects in each of the three treatment groups and 40 in the psychotherapy-alone sub-threshold dose condition. It might even be possible to use only 70 subjects

"Yet the political controversy about MDMA offers one crucial advantage that makes MDMA much more likely to become the first psychedelic to be approved as a prescription
medicine."

weeks, though longer-term follow-up data should also be gathered.

\section{Orphan Drug Designation: Not Possible}

per group, since Dr. Kazdin has estimated, "for comparing two treatments [for superiority, not equivalence, making this a high estimate for a test of equivalence]...a sample size of 71 per group would be needed to retain power at the desired level for the median ES [effect size]." (Kazdin \& Bass 1989).

\section{Duration of Studies}

The studies of Zoloft that Pfizer submitted for review were designed as 12-week trials. Dr. Marmar noted that "suicide rates are an important issue both in the acute and chronic form (Psychopharmacologic Drugs Advisory Committee 1999: 27)," suggesting caution in the use of placebo groups in PTSD patients with a risk factor for suicide. Relatively short treatment courses should be employed to minimize the amount of time patients are receiving placebo, or instead psychotherapy-alone with a subthreshold dose of MDMA, which will maximize suggestion without providing a direct pharmacological effect of MDMA.

Dr. Domingez, Advisory Committee Member, suggested that 12 weeks was sufficient for the study since most people respond by then. She noted that there was a trade-off between the desire to extend treatment in order to give enough time to find an effect and the desire not to keep people on placebo for an unnecessarily long period of time (Psychopharmacologic Drugs Advisory Committee 1999: 129).

This discussion supports limiting the length of MDMA treatment in the clinical trials to 12
Dr. Marmar stated that the lifetime prevalence for PTSD in the American adult population is $7.8 \%$. Dr. Bonnie Green, Professor of Psychiatry at Georgetown University Medical School, President Elect of the International Society for Traumatic Stress Studies (ISTSS) commented that any one time, $5 \%$ of women and $2-3 \%$ of men have PTSD (Psychopharmacologic Drugs Advisory Committee 1999: 22). Since the adult population of the United States is greater than 170 million, PTSD clearly does not qualify as an 0rphan disease since there are more than 200,000 potential patients in any given year.

\section{MAPS' Clinical Plan for MDMA for PTSD}

The following outline is of a sequence of studies designed to evaluate the risks and benefits of the use of MDMA-assisted psychotherapy in the treatment of post traumatic stress disorder (PTSD). This plan includes only studies focused on the safety and efficacy of the use of one to four sessions of MDMA-assisted psychotherapy in patients with PTSD. The Clinical Plan begins with a Phase II study since Phase I MDMA safety studies have already been conducted in the United States, Spain and Switzerland.

As the studies of MDMA in patients with PTSD are conducted, additional safety issues may become apparent. Further research addressing specific issues related to the safety of MDMA may be required by FDA before there will be sufficient information to justify a New Drug Application (NDA). These additional studies, if needed, may involve issues that will be addressed by gov- 
ernment-funded research teams around the world already working to assess questions of safety and mechanisms of action. Alternatively, these issues may need to become the subject of research by MAPS-funded scientific teams. However, based on what is already known about MDMA, it is likely that any safety issues related to the use of MDMA in PTSD patients can be adequately addressed by the proposed studies in PTSD patients.

\section{Phase II Spain Dose-Finding Pilot Study in Women Survivors of Sexual Assault}

This study, being conducted by Dr. Pedro Sopelano and Jose Carlos Bouso, Ph.D. candidate, U. Autonoma de Madrid, is currently the only MDMA psychotherapy study underway anywhere in the world in which MDMA is being administered to patients. The goals of this study are, 1) to evaluate whether a single dose of MDMA can be administered safely to 29 female survivors of sexual assault with chronic PTSD, 2) to gather preliminary evidence about therapeutic efficacy and, 3) to determine which dose or doses should be used in subsequent largerscale studies. This study treated the sixth subject on April 15, 2002 and is scheduled to complete the testing of all 29 subjects by May 2003.

The Phase II dose/response study in Spain will cost $\$ 65,000$, or $\$ 2,240$ per subject. The Spain study involves just one treatment session per subject. The study is being coordinated by Jose Carlos Bouso, a Ph.D. candidate working on the study for his dissertation. Under these circumstances, a cost of $\$ 2,240$ per subject can be obtained. This is the lower limit for the costper-patient of any MDMA protocol.

\section{Phase II United States Full-Dose Pilot Study in Male and Female PTSD patients}

$A$ research team under the director of $D r$. Michael Mithoefer has worked with MAPS to design and obtain FDA-approval to conduct an
MDMA/PTSD pilot study in the United States. The protocol was approved by the FDA on November 2, 2001. As of May 2002, the protocol is still in the midst of the IRB approval process. The study should begin Summer 2002. The protocol will involve 20 subjects with PTSD, both male and female. All 20 subjects will receive about 12 hours of non-drug psychotherapy. Twelve subjects will also receive two sessions of MDMA-assisted psychotherapy scheduled three to five weeks apart, with a dose of $125 \mathrm{mgs}$ at each session, while 8 subjects will receive 2 placebo sessions. The goals of this study are 1) to evaluate whether MDMA can be safely administered to PTSD patients and 2) to determine whether there is any preliminary evidence of therapeutic efficacy and, if so, to develop an estimate of the effect size.

The entire treatment course will be conducted in 12 weeks or less, in accordance with the recommendations made in the FDA Pharmacologic Drugs Advisory Committee meeting that reviewed the data from the trials of Zoloft in the treatment of PTSD,

If the study does begin in Summer 2002, the research team should be able to complete both sessions in all 20 patients by Summer 2003. The analysis of initial data can be completed by Fall 2003, with six month follow-up data analysis completed by Winter 2003. The final report can be completed by Spring 2004.

The cost of the study is estimated to be $\$ 12,000$ per subject or $\$ 240,000$. The costs of this study include non-drug psychotherapy hours as well as thorough neuropsychological evaluations, and quite a substantial cost for administrative work on the FDA and IRB approval process. Subsequent studies will probably require fewer non-drug psychotherapy hours and may not require any neuropsychological evaluations, depending on the results from this initial pilot study. Since administrative costs have been averaged over a small number of subjects, subsequent studies with much larger subject populations, at least 10 times the size of this pilot 
study, can be conducted with significantly less cost per patient.

\section{Phase III Trials - 4 -Arm Multi-Site Study, United States}

The goal of this study is to be one of the two primary FDA-required "adequate and wellcontrolled investigations" demonstrating safety and efficacy of the use of MDMA in patients with PTSD. Depending on the data from the pilot studies, the study will focus either on women, on men, or on both. The study will be designed with a psychotherapy-alone group receiving a subthreshold (placebo) dose of MDMA, a medium dose group, a full dose group and a Zoloft or Paxil comparison group.

The number of sessions will be titrated by agreement of patient and therapeutic team, with a maximum of 4 sessions within a 12 week period. This study will hopefully start in Spring 2004 and will take three years to conduct. The study will enroll approximately 280 subjects, 80 in each drug treatment group and 40 in the psychotherapy-alone group. Due to economies of scale, the study should be able to be conducted for about $\$ 8,000$ per subject, for a total cost of $\$ 2,240,000$.

\section{Phase III Trials- 4-Arm Study Spain or Israel}

The second large-scale trial will be conducted outside of the United States, in Spain or possibly in Israel. FDA will accept data gathered outside of the United States, if it is gathered according to standards set by FDA. With one study conducted in the United States and one in Spain or Israel, it should be possible to obtain marketing approval in both the United States and the European Community.

The goal of this study is to be one of the two primary "adequate and well-controlled investigations" demonstrating safety and efficacy. Depending on the data from the pilot studies, the study will focus either on women, on men, or on both. The study will be designed with a psychotherapy-alone group receiving a subthreshold (placebo) dose of MDMA, a medium dose group, a full dose group and a Zoloft or Paxil comparison group. The study will enroll approximately 280 subjects, 80 in each drug treatment group and 40 in the psychotherapyalone group. This study will involve a fixed number of sessions administered within a 12 week period. This study will involve three sessions for each subject, once every four weeks, with no titration permitted. The use of two different designs for the two different Phase III studies, with the US study using a variable number of treatment sessions depending upon patient and therapist decision and the foreign study employing a fixed number of three sessions, is based on the recommendation made by Dr. Hammer during the October 8, 1999 meeting of FDA's Pharmacologic Drugs Advisory Committee.

This study will hopefully start in Spring 2004 and will take three years to conduct. The study will enroll 280 subjects, should cost in the range of $\$ 8,000$ per subject, for $\$ 2,240,000$.

\section{Total Cost}

The total cost of the sequence of studies enumerated above amounts to $\$ 4,720,000$. Additional animal or human toxicity studies may be needed, though it is likely that these studies will have already been government-funded with the data in the public domain.

The Clinical Plan elaborated above suggests that a rough estimate of about $\$ 5$ million will need to be expended over a five-year period to develop MDMA into a prescription medicine for just one clinical indication, PTSD. After MDMA is approved initially for PTSD, only one adequate and well controlled multi-site investigation might be sufficient for the approval of subsequent uses of MDMA in closely related disorders, such as in the psychotherapeutic treatment of anxiety and depression in cancer patients. 


\section{References}

Baggott, M.; Jerome, I. \& Stuart R. 2001. 3,4methylenedioxymethamphetamine (MDMA)- a review of the English-language scientific and medical literature. Submitted to FDA as part of IND \# 63,384. MAPS website. http://www.maps.org/ research/mdma/protocol/litreview.html

Bolla, K.; McCann, U. \& Ricaurte, G. 1998. Impairment in abstinent MDMA ("Ecstasy") users. Neurology 51(6):1532-7.

Croft, R.; Mackay, A.; Mills, A. \& Gruzelier, J. 2001. The relative contributions of ecstasy and cannabis to cognitive impairment. Psychopharmacology (Berlin) 153(3): 373-9.

Cami, J.; Farre, M.; Mas, M.; Roset, P.; Poudevida, S.; Mas, A.; San, L. \& de la Torre, R. 2000. Human pharmacology of 3,4methylenedioxymethamphetamine ("ecstasy"): psychomotor performance and subjective effects. Journal of Clinical Psychopharmacology 20(4):455-66.

de la Torre, R.; Farre, M.; Ortuno, J.; Mas, M.; Brenneisen, R.; Roset, P.; Segura, J. \& Cami, J. 2000. Non-linear pharmacokinetics of MDMA ('ecstasy') in humans. British Journal of Clinical Pharmacology 49(2):104-9.

Doblin, R. 2001. The Regulation of the medical use of psychedelics and marijuana. Unpublished Public Policy doctoral dissertation, Kennedy School of Government, Harvard University. http:// www.maps.org/dissertation

Everhart, E.; Jacob III, P.; Shwonek, P.; Baggott, M.; Jones, R. \& Mendelson, J. 1999. Estimation of the Metabolic Disposition of MDMA and MDA Enantiomers in Humans. Abstracts - College on Problems of Drug Dependence (CPDD) Annual Meeting : 41.

Food and Drug Administration. 1998. Providing Clinical Evidence of Effectiveness for Human Drug and Biological Products. FDA Guidance for Industry. http://www.fda.gov/cder/guidance/ 1397fnl.pdf

Lieberman, J. \& Aghajanian, G. 1999. Caveat emptor: researcher beware. Neuropsychopharmacology 21(4):471-3.

Fallon, J.; Kicman, A.; Henry, J.; Milligan, P.; Cowan, D. \& Hutt, A. 1999. Stereospecific analysis and enantiomeric disposition of 3, 4-methylenedioxymethamphetamine (Ecstasy) in humans. Clinical Chemistry 45 (7):1058-69 (Published erratum appears in Clinical Chemistry 45(9):1585).

Fischer, C.; Hatzidimitriou, G.; Wlos, J.; Katz, J. \& Ricaurte, G. 1995. Reorganization of ascending $5-\mathrm{HT}$ axon projections in animals previously exposed to the recreational drug (+/-)3,4methylenedioxymethamphetamine (MDMA, "ecstasy"). Journal of Neuroscience 15(8):5476-85.

Friedman, L.; Furberg, C. \& Demets, D. 1985. The Fundamentals of Clinical Trials. 2nd Edition. St Louis: Mosby-Year Book.

Gamma, A. 2001. Does Ecstasy cause memory deficits. A review of studies of memory function in Ecstasy users. MAPS website http:/ /www.maps.org/research/mdma/mdmamemory.html Grob, C.; Poland, R.; Chang, L. \& Ernst, T. 1996. Psychobiologic effects of 3,4-methylenedioxymethamphetamine in humans: methodological considerations and preliminary observations. Behavior and Brain Research. 73 (1-2):103-7.

Gouzoulis-Mayfrank, E.; Daumann, J.; Tuchtenhagen, F.; Pelz, S.; Becker, S.; Kunert, H.; Fimm, B. \& Sass, H. 2000. Impaired cognitive performance in drug free users of recreational ecstasy (MDMA). Journal of Neurology, Neurosurgery and Psychiatry 68 (6):719-25.

Hatzidimitriou, G.; McCann, U. \& Ricaurte, G. 1999. Altered serotonin innervation patterns in the forebrain of monkeys treated with (+/-)3,4-methylenedioxymethamphetamine seven years previously: factors influencing abnormal recovery. Journal of Neuroscience 19(12):5096-5107.

Helmlin, H.; Bracher, K.; Bourquin, D.; Vonlanthen, D. \& Brenneisen, R. 1996. Analysis of 3,4methylenedioxymethamphetamine (MDMA) and its metabolites in plasma and urine by HPLC-DAD and GC-MS. Journal of Analytical Toxicology 20 (6):432-40.

Henry, J.; Fallon, J.; Kicman, A.; Hutt, A.; Cowan, D. \& Forsling, M. 1998 Low-dose MDMA ("ecstasy") induces vasopressin secretion. Lancet 351 (9118):1784.

Hernandez-Lopez, C.; Farre, M.; Roset, P.; Menoyo, E.; Pizarro, N.; Ortuno, J.; Torrens, M.; Cami, J. \& de La Torre R. 2002. 3,4methylenedioxymethamphetamine (ecstasy) and alcohol interactions in humans: psychomotor performance, subjective effects, and pharmacokinetics. Journal of Pharmacology and Experimental Therapeutics 300 (1):236-44.

Johnston, L.; O'Malley, P. \& Bachman, J. 2001. Rise in ecstasy use among American teens begins to slow. University of Michigan News and Information Services: Ann Arbor, MI.

Katz, R. 1999. Memorandum, December 6, 1999 from Director of Division of Neuropharmacological Drug Products to File, NDA 19839/S-026. Obtained from FDA through FOIA request, along with entire approval package for Zoloft.

Kazdin, A. \& Bass, D. 1989. Power to detect differences between alternative treatments in comparative psychotherapy outcome research. Journal of Consulting and Clinical Psychology 57 (1): 138-147.

Leshner, A. 2001. Club Drugs Aren't "Fun Drugs." NIDA website. http://www.drugabuse.gov/Published_Articles/fundrugs.html

Liechti, M.; Baumann, C.; Gamma, A. \& Vollenweider, F. 2000 Acute psychological effects of 3,4Methylenedioxymethamphetamine (MDMA, "Ecstasy") are attenuated by the serotonin uptake inhibitor Citalopram. Neuropsychopharmacology. 22(5): 513-521.

Malberg, J.; Sabol, K. \& Seiden, L. 1996. Co-administration of MDMA with drugs that protect against MDMA neurotoxicity produces different effects on body temperature in the rat. Journal of Pharmacology and Experimental Therapeutics 278 (1):258-67. 
Malberg, J. \& Seiden, L. 1998. Small changes in ambient temperature cause large changes in 3,4methylenedioxymethamphetamine (MDMA)-induced serotonin neurotoxicity and core body temperature in the rat. Journal of Neuroscience 18 (13): 5086-94.

Mas, M.; Farre, M.; de la Torre, R.; Roset, P.; Ortuno, J.; Segura, J. \& Cami, J. 1999. Cardiovascular and neuroendocrine effects and pharmacokinetics of 3, 4-methylenedioxymethamphetamine in humans. Journal of Pharmacology and Experimental Therapeutics 290 (1):136-45.

McCann, U.; Szabo, Z.; Scheffel, U.; Dannals, R.; Ricaurte, G. 1998. Positron emission tomographic evidence of toxic effect of MDMA ("Ecstasy") on brain serotonin neurons in human beings. Lancet 352(9138):1433-7.

Mertl, M. 2000. Ecstasy and the Brain: Club Drug Rants and Raves. Brain.com. April 11.

Mithoefer, M. \& Wagner, M. 2001. A human phase II study safety and efficacy of 3,4-methylenedioxymethamphetamine (MDMA)-assisted psychotherapy in the treatment of chronic posttraumatic stress disorder (PTSD). FDA IND \# 63,384. MAPS website www.maps.org/research/mdma/protocol/index.html

National Drug Intelligence Center. October 2000. Draft National Drug Threat Assessment 2001: The Domestic Perspective. http:/ /www.usdoj.gov/ndic/products.htm

National Institute on Drug Abuse (NIDA). 2000. Emerging Drug Epidemics: Club Drugs. NIDA website http://165.112.78.61/ Meetings/ClubSat.html

Office of Applied Studies. 2001a. Mortality Data from the Drug Abuse Warning Network-2000. Substance Abuse Mental Heath Services Administration. National Institutes of Health, Drug Abuse Warning Network. http://www.samhsa.gov/statistics/ statistics.html..

Office of Applied Studies. 2001b. Updated weighted emergency department estimates for the coterminous U.S.by year: 1994 to 2000. Substance Abuse Mental Heath Services Administration. National Institutes of Health, Drug Abuse Warning Network. (3/ 2001, update). http://www.samhsa.gov/oas/dawn.htm

Office of Public Affairs, United States Customs Service. January 5, 2000. Ecstasy Seizures and Smuggling Methods Fact Sheet.

Otalora M. 1984. MDMA and LSD therapy in the treatment of posttraumatic stress disorder in a case of sexual abuse. MAPS Website http://www.maps.org/research/mdma/marcela.html

Pacifici, R.; Zuccaro, P.; Farre, M.; Pichini, S.; Di Carlo, S.; Roset, P.; Ortuno, J.; Segura, J. \& de la Torre, R.1999. Immunomodulating properties of MDMA alone and in combination with alcohol: a pilot study. Life Sciences 65(26): PL309-16.

Pharmacologic Drugs Advisory Committee. October 8, 1999 meeting transcript. FDA website. http://www.fda.gov/ohrms/dockets/ac/cder99t.htm\#Psychopharmacologic\%20Drugs3556t1a.pdf
Reneman, L.; Lavalaye, J.; Schmand, B.; de Wolff, F.; , van den Brink, W.; den Heeten G, \& Booij, J. 2001. Cortical serotonin transporter density and verbal memory in individuals who stopped using 3,4-methylenedioxymethamphetamine (MDMA or "ecstasy"): preliminary findings. Archives of General Psychiatry 58 (10): 9016.

Ricaurte, G.; DeLanney, L.; Irwin, I.; Langston, J. 1988. Toxic effects of MDMA on central serotonergic neurons in the primate: importance of route and frequency of drug administration. Brain Research 446 (1):165.

Smith, D. 1999. Sertraline for PTSD: NDA 19-839. FDA Website http://www.fda.gov/ohrms/dockets/ac/99/slides/3556s1a/ sld001.htm

Sopelana, P. \& Bouso, J. 1999. Administration of 3,4methylenedioxymethamphetamine (MDMA) to women with chronic Posttraumatic Stress Disorder (PTSD) as a consequence of sexual assault: a dose-finding pilot study. Spanish Drug Agency Code \# 99-0309. MAPS Website www.maps.org/research/mdma/spain/ index.html

Stevens, S. 1999/2000. Speaking the silence: MDMA in a couple dealing with cancer. Bulletin of the Multidisciplinary Assocation for Psychedelic Studies, 9 (4): 31-34. http://www.maps.org/newsletters/v09n4/09431sue.html

Tancer, M. \& Johanson CE. The subjective effects of MDMA and mCPP in moderate MDMA users. Drug and Alcohol Dependence 2001 Dec 1;65(1):97-101.

Vollenweider, F.; Gamma, A.; Liechti, M. \& Huber, T. 1998. Psychological and cardiovascular effects and short-term sequelae of MDMA ("ecstasy") in MDMA-naive healthy volunteers. Neuropsychopharmacology 19(4): 241-51.

Vollenweider, F.; Gamma, A.; Liechti, M. \& Huber, T. 1999a. Is a single dose of MDMA harmless? Neuropsychopharmacology 21(4): 598-600.

Vollenweider, F.; Remensberger, S.; Hell, D. \& Geyer, M. 1999b. Opposite effects of 3,4-methylenedioxymethamphetamine (MDMA) on sensorimotor gating in rats versus healthy humans. Psychopharmacology (Berlin) 143 (4): 365-72.

Vollenweider, F. 2001. Personal communication. 9/14/01.

Zakzanis, K. \& Young, D. 2001. Memory impairment in abstinent MDMA ("Ecstasy") users: a longitudinal investigation. Neurology 56(7):966-9. 